\title{
Evaluating the clinical significance of SHMT2 and its co-expressed gene in human kidney cancer
}

Huan Wang ${ }^{1,2,3}$, Tie Chong ${ }^{1 *}$, Bo-Yong Li ${ }^{2,3}$, Xiao-San Chen ${ }^{2,3}$ and Wen-Bo Zhen ${ }^{2,3}$

\begin{abstract}
Background: Kidney cancer is one of the most common cancers in the world. It is necessary to clarify its underlying mechanism and find its prognostic biomarkers. Current studies showed that SHMT2 may be participated in several kinds of cancer.

Methods: Our studies investigated the expression of SHMT2 in kidney cancer by Oncomine, Human Protein Atlas database and ULCAN database. Meanwhile, we found its co-expression gene by cBioPortal online tool and validated their relationship in A498 and ACHN cells by cell transfection, western blot and qRT-PCR. Besides these, we also explored their prognostic values via the Kaplan-Meier plotter database in different types of kidney cancer patients.

Results: SHMT2 was found to be increased in 7 kidney cancer datasets, compared to normal renal tissues. For the cancer stages, ages and races, there existed significant difference in the expression of SHMT2 among different groups by mining of the UALCAN database. High SHMT2 expression is associated with poor overall survival in patients with kidney cancer. Among all co-expressed genes, NDUFA4L2 and SHMT2 had a high co-expression efficient. SHMT2 overexpression led to the increased expression of NDUFA4L2 at both MRNA and protein levels. Like SHMT2, overexpressed NDUFA4L2 also was associated with worse overall survival in patients with kidney cancer.
\end{abstract}

Conclusion: Based on above results, overexpressed SHMT2 and its co-expressed gene NDUFA4L2 were all correlated with the prognosis in kidney cancer. The present study might be benefit for better understanding the clinical significance of SHMT2 and provided a potential therapeutic target for kidney cancer in future.

Keywords: SHMT2, NDUFA4L2, Prognosis, Kidney cancer, Bioinformatic analysis

\section{Background}

Kidney cancer, as one of the most common cancers in the world, is becoming a severe global burden but lacks public attention [1]. Among various types of kidney cancer, clear cell renal cell carcinoma (ccRCC) was the most common subtype of kidney cancer, accounting for more than $85 \%$ of all kidney cancer [2]. Despite great achievement in diagnosis and therapy, because of the

*Correspondence: chongtie001@hotmail.com

${ }^{1}$ Department of Urology, Second Affiliated Hospital of Medical School, Xi'an Jiaotong University, Xi'an, Shaanxi 710061, P.R. China

Full list of author information is available at the end of the article characteristics of high metastasis risk and poor response to radiotherapy and chemotherapy, the most of patients with advanced kidney cancer are still difficult to be cured and prolong the survival time [3]. Therefore, it is necessary to clarify the underling mechanism and find several clinically effective diagnostic and prognostic biomarkers to prevent its occurrence and re-occurrence.

Serine Hydroxymethyltransferase 2 (SHMT2), a protein coding gene, regulate glycine production in mitochondria, which is an essential intermediate for purine biosynthesis $[4,5]$. In general, this gene is over-expressed in liver, lymph node and peripheral blood mononuclear cells. Nevertheless, it has been reported to a valuable

(c) The Author(s) 2020. This article is licensed under a Creative Commons Attribution 4.0 International License, which permits use, sharing, adaptation, distribution and reproduction in any medium or format, as long as you give appropriate credit to the original author(s) and the source, provide a link to the Creative Commons licence, and indicate if changes were made. The images or other third party material in this article are included in the article's Creative Commons licence, unless indicated otherwise in a credit line to the material. If material is not included in the article's Creative Commons licence and your intended use is not permitted by statutory regulation or exceeds the permitted use, you will need to obtain permission directly from the copyright holder. To view a copy of this licence, visit http://creativeco mmons.org/licenses/by/4.0/. The Creative Commons Public Domain Dedication waiver (http://creativecommons.org/publicdomain/ zero/1.0/) applies to the data made available in this article, unless otherwise stated in a credit line to the data. 
marker in several cancers, including intrahepatic cholangiocarcinoma [6], large B cell lymphoma [7] and breast cancer [8]. Besides these studies, the mitochondrial onecarbon metabolic pathway involving in SHMT2 is also associated with patient survival in pancreatic cancer and gastrointestinal cancer $[9,10]$. However, current studies have not focused on the relationship between SHMT2 and kidney cancer. And its expression profile and clinical significance of SHMT2 in human kidney cancer have not yet been investigated.

In our studies, we tried to explore the expression and prognosis of SHMT2 and its co-expression gene in kidney cancer for the first time by using multiple bioinformatics database. After experimental validation, we hoped that the assessment of both SHMT2 and its co-expressed gene in kidney cancer will be helpful in predicting kidney cancer prognosis and providing a novelty therapeutic target for individual treatment of kidney cancer.

\section{Materials and methods Ethics statement}

No human or animal specimens were utilized in our studies. And all the datasets were acquired from the public databases and articles, which all met with the Declaration of Helsinki.

\section{Oncomine database}

Oncomine database (https://www.oncomine.org/), a cancer database bioinformatics tool and online datamining platform, was used to explore the mRNA levels of SHMT2 and NADH dehydrogenase [ubiquinone] 1 alpha sub complex, 4-like 2 (NDUFA4L2) in kidney cancer. We searched the database for the fold changes of SHMT2 and NDUFA4L2 in kidney cancer using the filters of differential analysis (cancer vs normal), cancer type (kidney cancer), sample type (clinical specimen), data type (mRNA), and gene (SHMT2 and NDUFA4L2). Students' t-test was used to generate a $\mathrm{p}$ value. To obtain the most significant SHMT2 and NDUFA4L2probes, we set the following parameters: the cut-off of $\mathrm{p}$ value and fold change were defined as 0.01 and 2 , respectively.

\section{GEPIA database}

Gene Expression Profiling Interactive Analysis (GEPIA) (http://gepia.cancer-pku.cn) database, is a newly developed interactive web server developed by Zefang Tang, Chenwei Li and Boxi Kang of Zhang Lab, Peking University, focused on scrutinizing the RNA sequencing expression data of 9,736 tumors and 8,587 normal samples from the TCGA and the GTEx projects, using a standard processing pipeline. In this study, we used GEPIA to validate the expression levels of SHMT2. We set the following parameters: the cut-off of $\mathrm{p}$ value and fold change were defined as 0.01 and 2 , respectively.

\section{Human Protein Atlas database}

Human Protein Atlas (http://www.proteinatlas.org) is a Swedish-based program initiated in 2003 with the aim to map all the human proteins in cells, tissues and organs using integration of various omics technologies. In our study, the Human Protein Atlas was used for immunohistochemistry (IHC) validation of several candidate genes SHMT2 and NDUFA4L2.

\section{UALCAN database}

The UALCAN web portal (ualcan.path.uab.edu/) has an important feature that aids querying based on the gene class. Meanwhile, it is a user-friendly, interactive web resource for analyzing cancer transcriptome data. Using UALCAN database, we analyzed the expression profiles of SHMT2 and NDUFA4L2 in normal and renal clear cell carcinoma samples based on clinicopathologic parameters, such as cancer stage, age, race, and tumor grade.

\section{cBioPortal database}

The cBioPortal (www.cbioportal.org/) for Cancer Genomics was originally developed at Memorial Sloan Kettering Cancer Center (MSK), which contained both sequencing and pathological data on 30 different cancers. The kidney cancer (TCGA, renal clear cell carcinoma; Provisional) dataset including data from 538 samples with pathology reports was selected for further analyses of SHMT2.

\section{Cell culture and transfection}

Human renal carcinoma cell lines A498 and ACHN cells were purchased from American Type Culture Collection (ATCC, Manassas, VA, USA). The cells were cultured in minimum essential medium (MEM, Hyclone) supplemented with $10 \%$ fetal bovine serum (FBS). All cells were maintained in a humidified incubator with $5 \% \mathrm{CO}_{2}$ at $37^{\circ} \mathrm{C}$. Human SHMT2 cDNA ORF clone (cat. RC204239) and the empty control pCMV6-AC (cat. PS100020) were obtained from OriGene (Rockville, USA). A498 and ACHN cells were transfected with the expression vector or empty control using Lipofectamine 3000 (Life Technologies, CA, USA).

\section{qRT-PCR}

Total cell RNAs were extracted utilizing the Trizol Reagent (Invitrogen, CA, USA). Then, the RNA samples were reverse-transcribed using the iScript cDNA Synthesis kit (Bio-Rad, CA, USA) following the manufacturer's protocol. The primers of reference gene ( $\beta$-actin) and NDUFA4L2 were designed by Primer 5 software 
and synthesized by Shanghai Shenggong Bioengineering after BLAST comparison. Then, NDUFA4L2 mRNA was measured using qRT-PCR analysis with the following primers (sense:, 5'-TAATACGACTCACTATAG GG-3' and antisense: $5^{\prime}$ - TAGAAGGCACAGTCGAGG $\left.-3^{\prime}\right)$. The reference gene is $\beta$-actin, sense: $5^{\prime}$-TTGTAA CCAACT GGGACGATATGG- ${ }^{\prime}$, and antisense: $5^{\prime}$-GAT CTTGATCTTCATGGTGCTAG- ${ }^{\prime}$ And the real-time RT-PCR assays were performed using the 7500 Fast RealTime PCR System for quantitative mRNA detection and with iTaq Fast SYBR Green Supermix (Bio-Rad, Hercules, CA). The expression of genes in all groups was calculated using the 2- $\Delta \Delta \mathrm{Ct}$ method. All experiments were repeated three times independently.

\section{Western blot}

Total proteins were separated by SDS-PAGE after denaturation and transferred onto polyvinylidenedifluoride (PVDF) membranes. After blocking with 5\% skim milk, the membranes were incubated with rabbit anti-mouse monoclonal antibodies against. SHMT2 (1:300; Abcam, Cambridge, UK), NDUFA4L2 (1:1,000, Abcam, Cambridge, UK) and GAPDH(1:1000, Abcam, Cambridge, UK) overnight at $4{ }^{\circ} \mathrm{C}$ with shaking. Then, the membranes were washed in TBST. After that the membranes were washed and incubated with secondary antibody anti-rabbit IgG (1:2000, Santa Cruz, CA, USA) for $1.5 \mathrm{~h}$ at room temperature. Data obtained from the western blot experiments were analysed by Bio-Rad Quantity One 1D Analysis software (Bio-Rad, Hercules, CA, USA).

\section{Statistical analysis}

Data from western blot and qRT-PCR were presented in the form of mean \pm standard deviation (SD), and each experiment was independently repeated $\geq 3$ times. Statistical analyses were performed using Graphpad Prism 5 (Graphpad software Inc, San Diego, CA, USA). Data were analyzed for statistical significance by Student's t-test. p-value $<0.05$ was considered statistically significant.

\section{Results}

Expression levels of SHMT2 in patients with kidney cancer Utilizing the Oncomine database, we discovered the expression levels of SHMT2 in different kinds of kidney cancer with those in control samples. SHMT2 was upregulated in 7 various kidney cancer datasets, compared to normol tissues (P-value $<0.05$ and Fold change $>2$ ) including Renal Wilms Tumor [11], Clear Cell Renal Cell Carcinoma [11-13], Non-Hereditary Clear Cell Renal Cell Carcinoma [14] and Hereditary Clear Cell Renal Cell
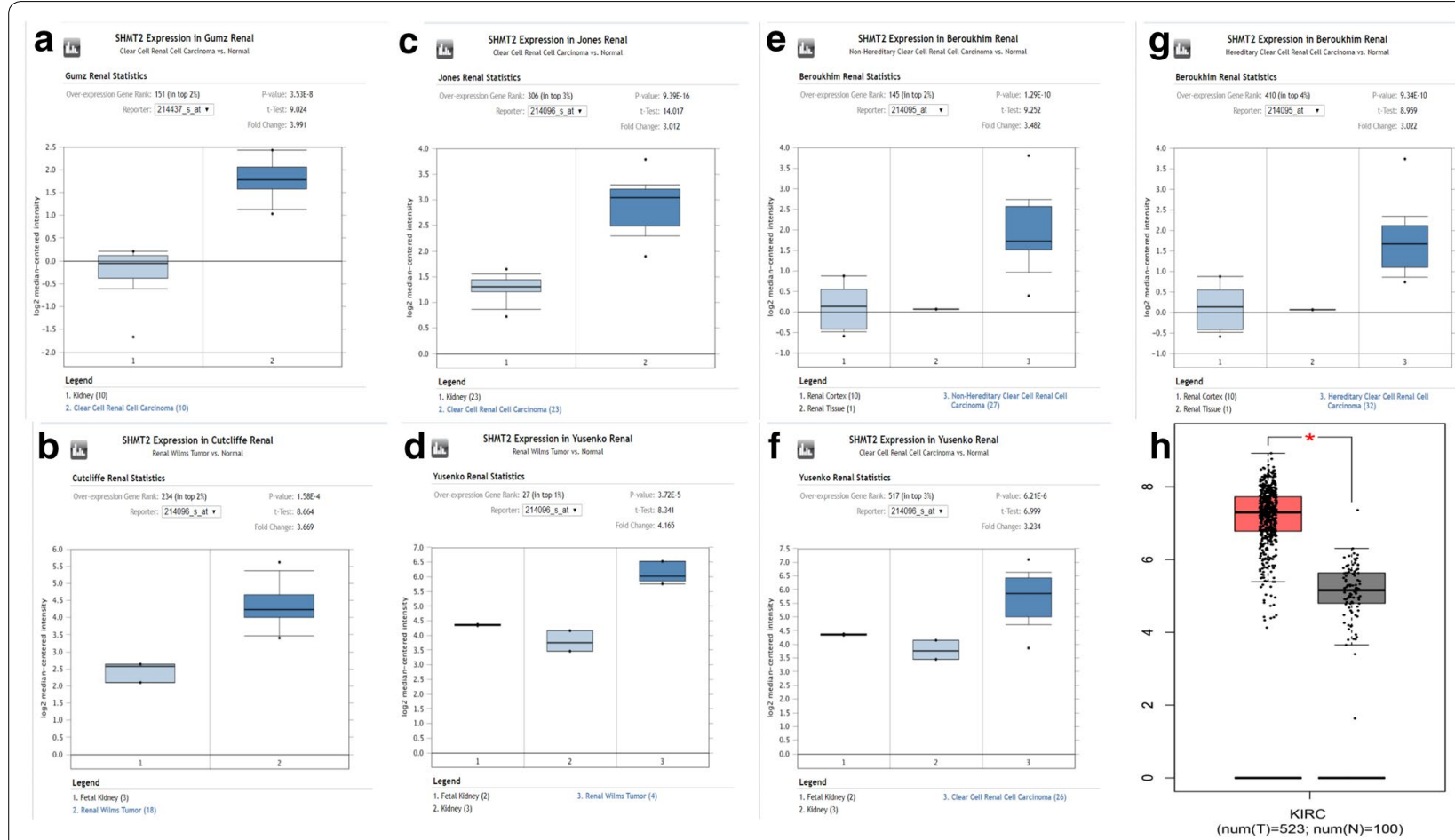

Fig. 1 the expression level of SHMT2 mRNA in human different types of kidney cancer a-g The expression of SHMT2 in different types of kidney cancer datasets from Oncomine database; $\mathbf{h}$ The expression of SHMT2 in renal clear cell carcinoma patients from GEPIA database. A total of 523 renal clear cell carcinoma tissues (red bar) and 100 normal renal tissues (gray bar) were collected in this database 
Carcinoma [14] (Fig. 1a-g). The comparisons of mRNA levels of SHMT2 in kidney cancer and healthy samples in each individual dataset were performed by using the Student's t-test. In addition, we also confirmed that mRNA expression levels of SHMT2 was significantly increased in renal clear cell carcinoma tissues than in normal renal tissues, which further verified the results from GEPIA database (Fig. 1h). A total of 523 renal clear cell carcinoma tissues (red bar) and 100 normal renal tissues (gray bar) were collected in this database.

\section{Association of SHMT2 expression with clinicopathological features from patients with renal clear cell carcinoma}

As shown in Fig. 2, we explored the expression of SHMT2 in normal and renal clear cell carcinoma tissues based on clinicopathologic parameters, such as cancer stage, age, race, and tumor grade by using UALCAN database. Our results indicated that SHMT2 was overexpressed in age (41-60 years) and (61-80 years) group compared to age (21-40 years) group $(\mathrm{P}<0.05)$. In term of the cancer stages, SHMT2 was higher in Stage 3 compared to the Stage $2(\mathrm{P}<0.05)$. And for races, the expression level of
SHMT2 was higher in Caucasian patients compared to the African-American patients $(\mathrm{P}<0.05)$. In regard to the tumor grade and patients' gender, there existed no significant difference in the expression of SHMT2 among different groups (Fig. 2).

\section{High SHMT2 expression is associated with poor OS of patients with kidney cancer}

Using the Kaplan-Meier plotter, the prognostic values of SHMT2 in various kidney cancers were predicted. As shown in Fig. 3b, we found that overexpressed SHMT2 is associated with worse overall survival (OS) patients with renal clear cell carcinoma, with $\mathrm{P}=0.033$ and $\mathrm{HR}=1.41$ (1.03-1.94). For patients with renal papillary cell carcinoma, we found that high expressedSHMT2 are also correlated with poor overall survival compared to those with low expression, with $\mathrm{P}=0.0024, \mathrm{HR}=2.45$ (1.35-4.44), using the median as the cutoff value (Fig. 3a). Subsequently, to confirm the predictive results, we investigated the IHC pictures of kidney cancer by using the HPA database. The IHC pictures validated that the SHMT2 showed a strongly intensity in kidney cancers compared with those in healthy samples (Fig. 3c, d).
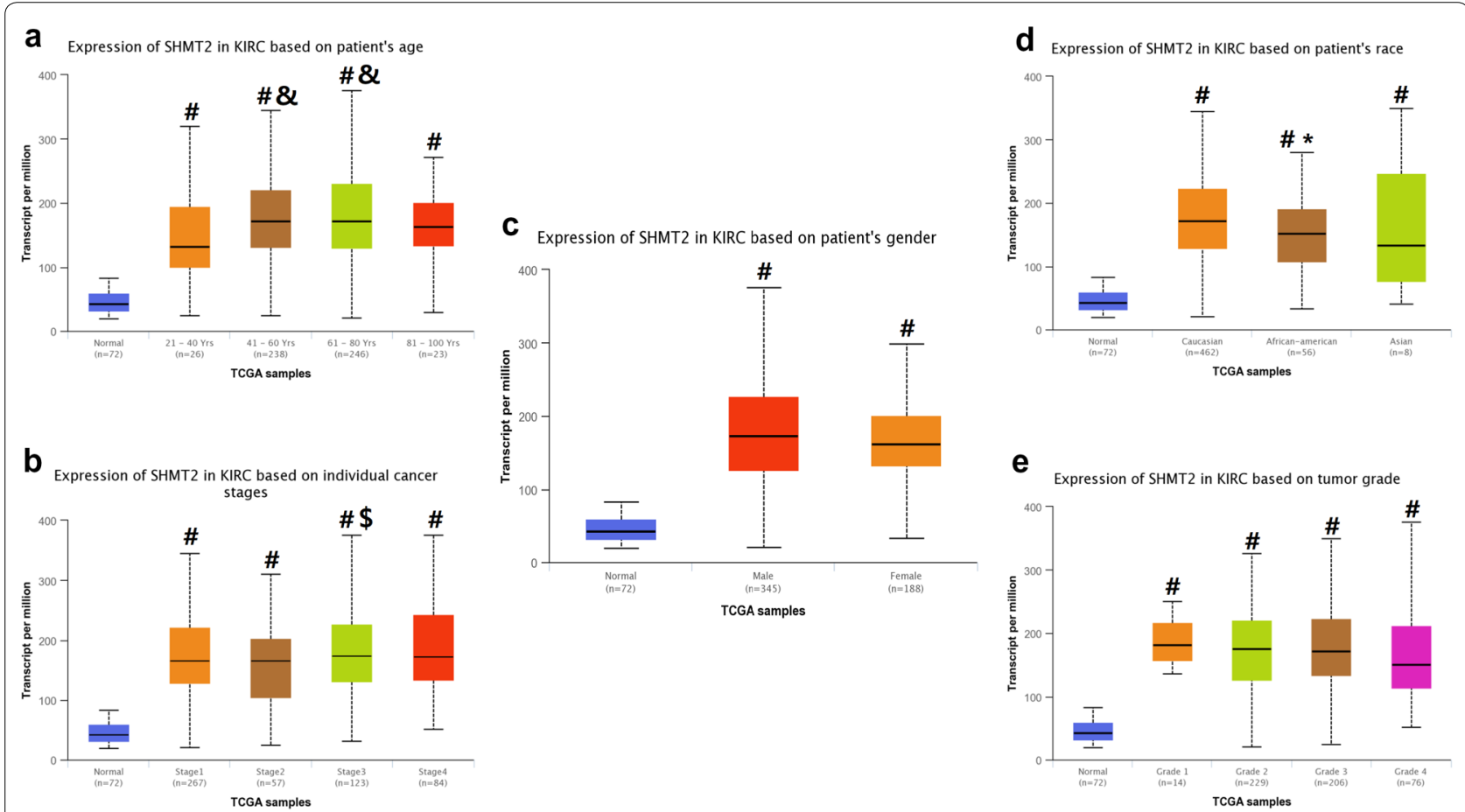

Fig. 2 Association between SHMT2 gene expression and clinical pathological parameters in patients with renal clear cell carcinoma (UALCAN). a expression of SHMT2 in normal and renal clear cell carcinoma tissues based on patients'age; $\mathbf{b}$ expression of SHMT2 in normal and renal clear cell carcinoma tissues based on cancer stages; c expression of SHMT2 in normal and renal clear cell carcinoma tissues based on patients' gender; d expression of SHMT2 in normal and renal clear cell carcinoma tissues based on patients' race; e expression of SHMT2 in normal and renal clear cell carcinoma tissues based on tumor grade. \#P:compared to normal group; \&P: compared to the age (21-40 years) group; \$P: compared to the Stage 2 group; *P: compared to the Caucasian group 


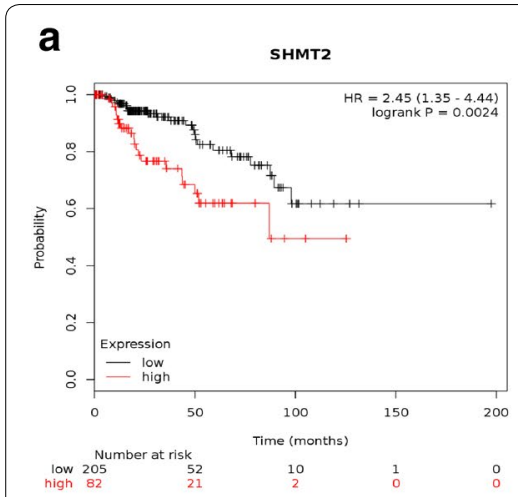

b SHMT2

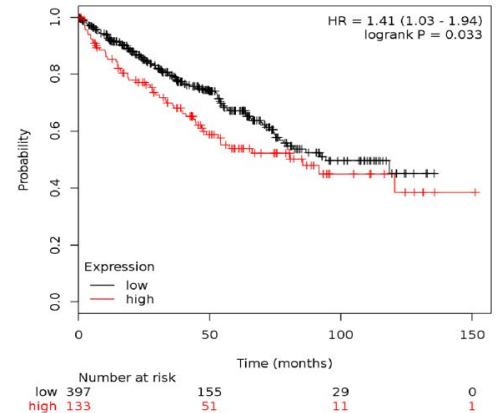

C
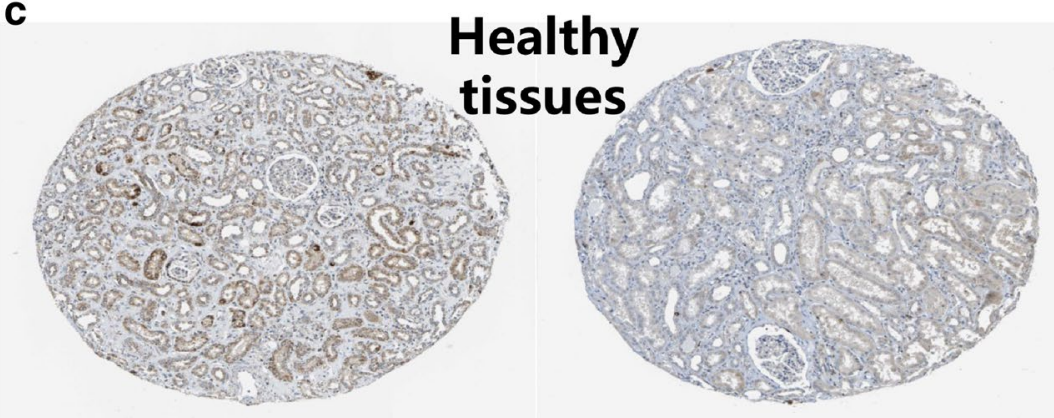

d

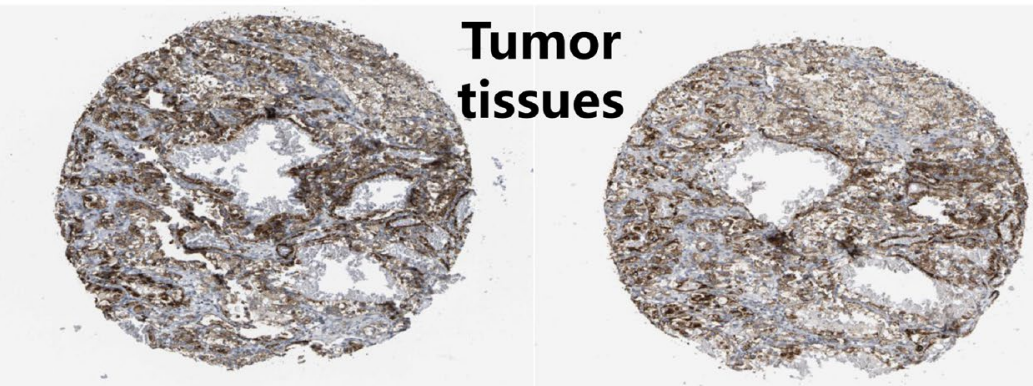

Fig. 3 The prognostic value of SHMT2 mRNA in kidney cancer patients (K-M plotter) and the IHC of SHMT2 in kidney cancer (HPA). $\mathbf{a}$, $\mathbf{b}$ Survival curve of SHMT2 for renal papillary cell carcinoma and renal clear cell carcinoma, respectively. $\mathbf{c}, \mathbf{d}$ : IHC of SHMT2 in kidney cancer from healthy and pathological tissues

\section{Analysis of SHMT2's co-expressed genes in kidney cancer} By examining the microarray data from cBioPortal online database for renal clear cell carcinoma (TCGA, Provisional), we identified the top 10 genes with positive correlation to SHMT2 expression in renal clear cell carcinoma, including NDUFA4L2, TCEA3, RACK1, HYI, LRRC23, RPLP0, ALDOA, PHB2, UBXN6 and RPS5 (Table 1). Among these co-expressed genes, NDUFA4L2 and SHMT2 had a high co-expression efficient, with Pearson's Correlation $=0.61$ (Fig. 4a). Besides, we also noticed that NDUFA4L2 is a newly biomarker that maybe involved in renal clear cell carcinoma occurrence and progression. To confirm the association between NDUFA4L2 and SHMT2, A498 and ACHN cells were transfected with SHMT2 expression vectors (Fig. 4b-d). In Fig. 4b, we detected SHMT2's expression in A498 and ACHN cells $24 \mathrm{~h}$ after transfection of SHMT2 expression vector or the negative control by western blot. The protein expression of SHMT2 was higher in SHMT2 expression vector group. In Fig. 4c, we found that SHMT2 overexpression could lead to significantly increased NDUFA4L2 expression at mRNA level by qRT-PCR. At the protein levels, NDUFA4L2's expression was also higher in A498 and
ACHN cells $24 \mathrm{~h}$ after transfection of SHMT2 expression vector (Fig. 4d).

\section{Expression levels of NDUFA4L2 in patients with kidney cancer and prognostic analysis}

To further analyze NDUFA4L2 expression in kidney cancer and normal renal tissues, we explored NDUFA4L2's expression in the above databases. In Oncomine database, NDUFA4L2 was also increased in 5 various kidney cancer datasets, compared to normol tissues (P-value $<0.05$ and Fold change $>2$ ) including Clear Cell Renal Cell Carcinoma, Non-Hereditary Clear Cell Renal Cell Carcinoma and Hereditary Clear Cell Renal Cell Carcinoma (Fig. 5). In UALCAN database, we found that NDUFA4L2 was higher in Stage 3 compared to the Stage 2 and Stage $1(\mathrm{P}<0.05)$. However, in respect of gender, races tumor grade and age, there is no significant difference in expression of SHMT2 among different groups (Fig. 6). Like SHMT2, overexpressed NDUFA4L2 also was associated with worse OS in patients with renal clear cell carcinoma and renal papillary cell carcinoma $(\mathrm{P}<0.05$, respectively) (Fig. 7). 
Table 1 Co-expression genes of SHMT2 in kidney cancer by cBioPortal

\begin{tabular}{lllllr}
\hline Correlated Gene & Cytoband & Pearson's Correlation & Spearman's Correlation & P-Value & Q-Value \\
\hline NDUFA4L2 & $12 q 13.3$ & 0.61 & 0.487509244 & $5.29 \mathrm{E}-28$ & $2.13 \mathrm{E}-24$ \\
TCEA3 & $1 \mathrm{p} 36.12$ & 0.55 & 0.536958268 & $1.13 \mathrm{E}-34$ & $1.11 \mathrm{E}-26$ \\
RACK1 & $5 \mathrm{q} 35.3$ & 0.54 & 0.476685233 & $1.04 \mathrm{E}-28$ & $7.19 \mathrm{E}-23$ \\
HYI & $1 \mathrm{p} 34.2$ & 0.51 & 0.493135371 & $4.03 \mathrm{E}-26$ & $8.13 \mathrm{E}-23$ \\
LRRC23 & $12 \mathrm{p} 13.31$ & 0.51 & 0.471964961 & $5.59 \mathrm{E}-26$ & $1.03 \mathrm{E}-22$ \\
RPLP0 & $12 \mathrm{q} 24.23$ & 0.51 & 0.470753952 & $2.76 \mathrm{E}-28$ & $1.39 \mathrm{E}-24$ \\
ALDOA & $16 \mathrm{p} 11.2$ & 0.5 & 0.489777334 & $3.22 \mathrm{E}-33$ & $3.25 \mathrm{E}-29$ \\
PHB2 & $12 \mathrm{p} 13.31$ & 0.49 & 0.526836541 & $3.39 \mathrm{E}-25$ & $5.70 \mathrm{E}-22$ \\
UBXN6 & $19 \mathrm{p} 13.3$ & 0.46 & 0.464023674 & $2.97 \mathrm{E}-26$ & $6.66 \mathrm{E}-23$ \\
RPS5 & $19 \mathrm{q} 13.43$ & 0.45 & 0.473084127 & &
\end{tabular}

a

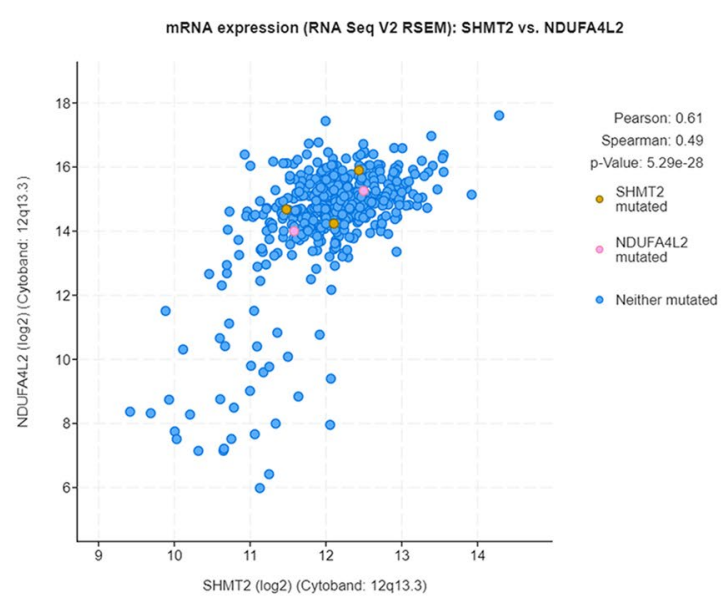

b

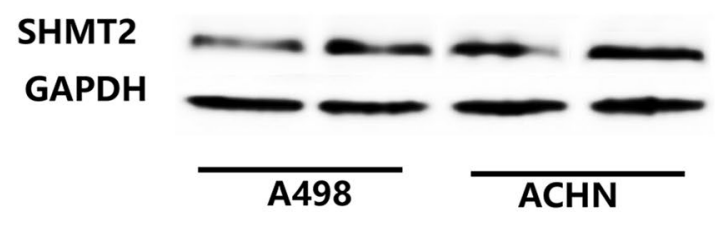

C

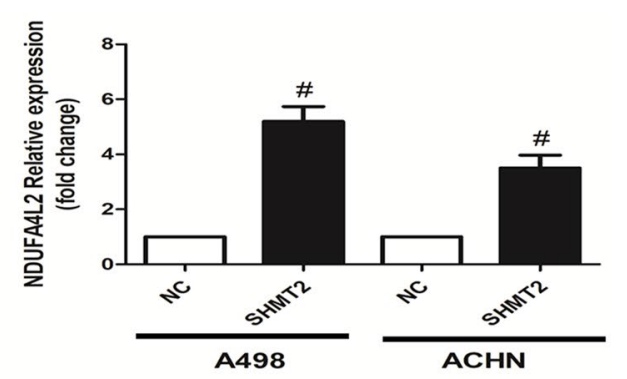

d

NC SHMT2 NC SHMT2
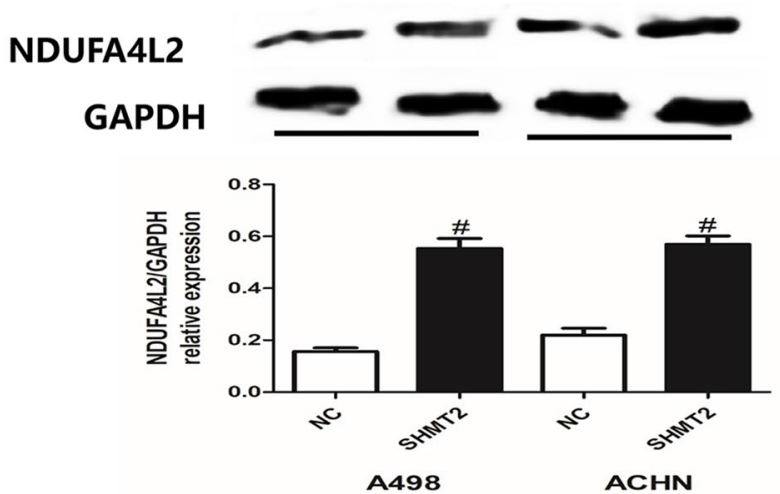

Fig. 4 Analysis of SHMT2's co-expressed genes in kidney cancer a regression analysis of the expression between SHMT2 and NDUFA4L2 in kidney cancer by using cBioPortal tool. b Western blot analysis and representative image of SHMT2 expression in A498 and ACHN cells $24 \mathrm{~h}$ after transfection of SHMT2 expression vector or the negative control. c NDUFA4L2 expression at mRNA level in A498 and ACHN cells $24 \mathrm{~h}$ after transfection of SHMT2 expression vector or the negative control. $\mathbf{d}$ Western blot assay and representative results of NDUFA4L2 expression at protein level in A498 and ACHN cells $24 \mathrm{~h}$ after transfection of SHMT2 expression vector or the negative control. $n=3$; \#p $<0.05$, vs negative control

\section{Discussion}

In current research, we found that SHMT2 is overexpressed in different kinds of kidney cancer with those in control samples. Moreover, we also analyzed the correlation of SHMT2 expression with clinicopathological features from patients with renal clear cell carcinoma. As a protein coding gene, SHMT2 also possessed prognostic value for renal clear cell carcinoma, which was also validated by immunohistochemical staining from pathological tissues and healthy tissues. Then Analysis of SHMT2's co-expressed genes in kidney cancer discovered that NDUFA4L2 was the primary co-expressed gene with SHMT2 in kidney cancer. Through experimental verification, we confirmed that SHMT2 overexpression could 


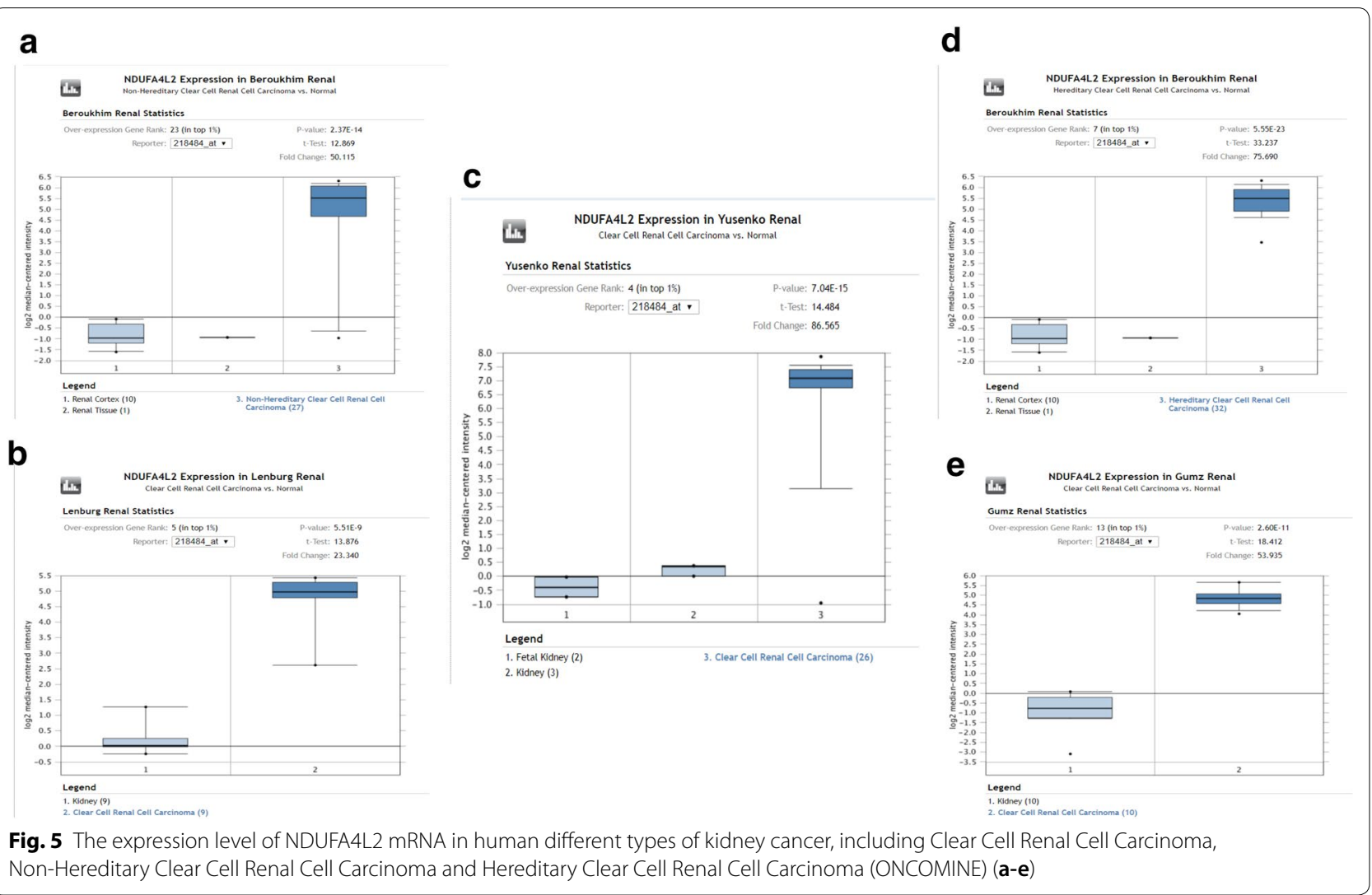

lead to significantly increased NDUFA4L2 expression at both mRNA and protein levels in A498 and ACHN cells. After data mining by multiple bioinformatic tools, these results suggested that NDUFA4L2 was also high expressed in various types of kidney cancer and was associated with worse OS in patients with renal clear cell carcinoma, like SHMT2. Our studies provided a novelty biomarker and analyzed its significant clinical relevance in kidney cancer.

SHMT2 encodes the mitochondrial form of a pyridoxal phosphate-dependent enzyme that catalyzes the reversible reaction of serine and tetrahydrofolate to glycine and 5,10-methylene tetrahydrofolate. The encoded product is primarily responsible for glycine synthesis. Current studies showed that SHMT2 may be participated in several kinds of cancers, such as breast cancer $[8,15]$, glioma [16], intrahepatic cholangiocarcinoma [6] and colorectal cancer [17]. Besides these studies, a recent research also reported that SHMT2 desuccinylation is a pivotal signal in cancer cells to adapt serine metabolic processes for rapid growth. To prevent the tumor growth and proliferation, SIRT5 could be a potential therapeutic target for inhibiting serine catabolism. A meta-analysis also found that SHMT2 and its downstream enzyme MTHFD2 were broadly required for cancer cell proliferation and viability [18]. Through proteomic profiling of breast cancer metabolism, Bernhardt et al. identified the importance of SHMT2 and ASCT2 in breast cancer patients, which could serve as valuable individual prognostic markers and potential targets and may beneficial to personalized breast cancer therapy [8]. In neuroblastoma patient samples, there was a significant correlation between SHMT2 and hypoxia-inducible factor-1 $\alpha$ (HIF-1 $\alpha$ ), and SHMT2 expression correlated with worse patient prognosis [19]. As for colorectal cancer, Wei et al. demonstrated that SHMT2 is acetylated at K95 in colorectal cancer cells. However, as the major deacetylase in mitochondria, SIRT3 is responsible for SHMT2 deacetylation. Their studies showed that deacetylation of SHMT2 by SIRT3 could enhance colorectal carcinogenesis and was correlated with poorer postoperative overall survival [20]. And a recent publication also showed that SHMT2 is overexpressed in kidney cancers, particulay in the high stage tumors [19]. Our investigations may helpful to further fill in the blank and understand the biological significance of SHMT2 in kidney cancer.

Through co-expression analysis by cBioPortal database, we identified a SHMT2's co-expressed gene, NDUFA4L2, in kidney cancer. NDUFA4L2's related pathway included in respiratory electron transport, ATP synthesis by 
a. Expression of NDUFA4L2 in KIRC based on individual cancer

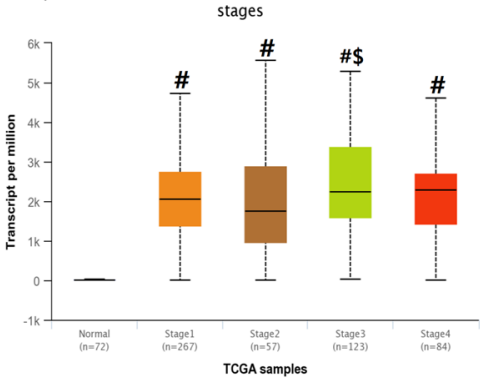

D Expression of NDUFA4L2 in KIRC based on patient's age

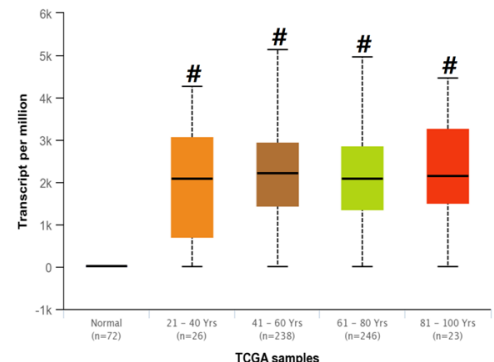

\section{C}

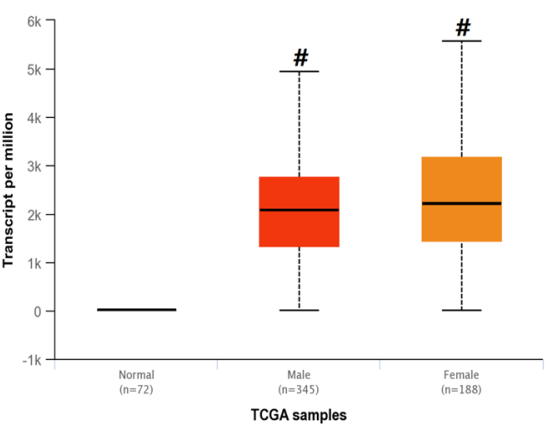

d Expression of NDUFA4L2 in KIRC based on patient's race

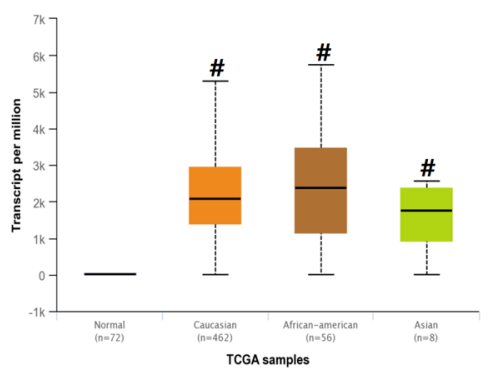

e

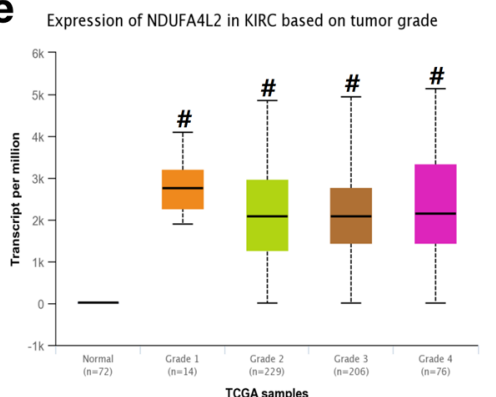

Fig. 6 Association between NDUFA4L2 gene expression and clinical pathological parameters in patients with renal clear cell carcinoma (UALCAN). a expression of NDUFA4L2 in normal and renal clear cell carcinoma tissues based on cancer stages; $\mathbf{b}$ expression of SHMT2 in normal and renal clear cell carcinoma tissues based on patients' age; c expression of SHMT2 in normal and renal clear cell carcinoma tissues based on patients' gender; $\mathbf{d}$ expression of SHMT2 in normal and renal clear cell carcinoma tissues based on patients'race; e expression of SHMT2 in normal and renal clear cell carcinoma tissues based on tumor grade. \#P:compared to normal group; \$P: compared to the Stage 2 group

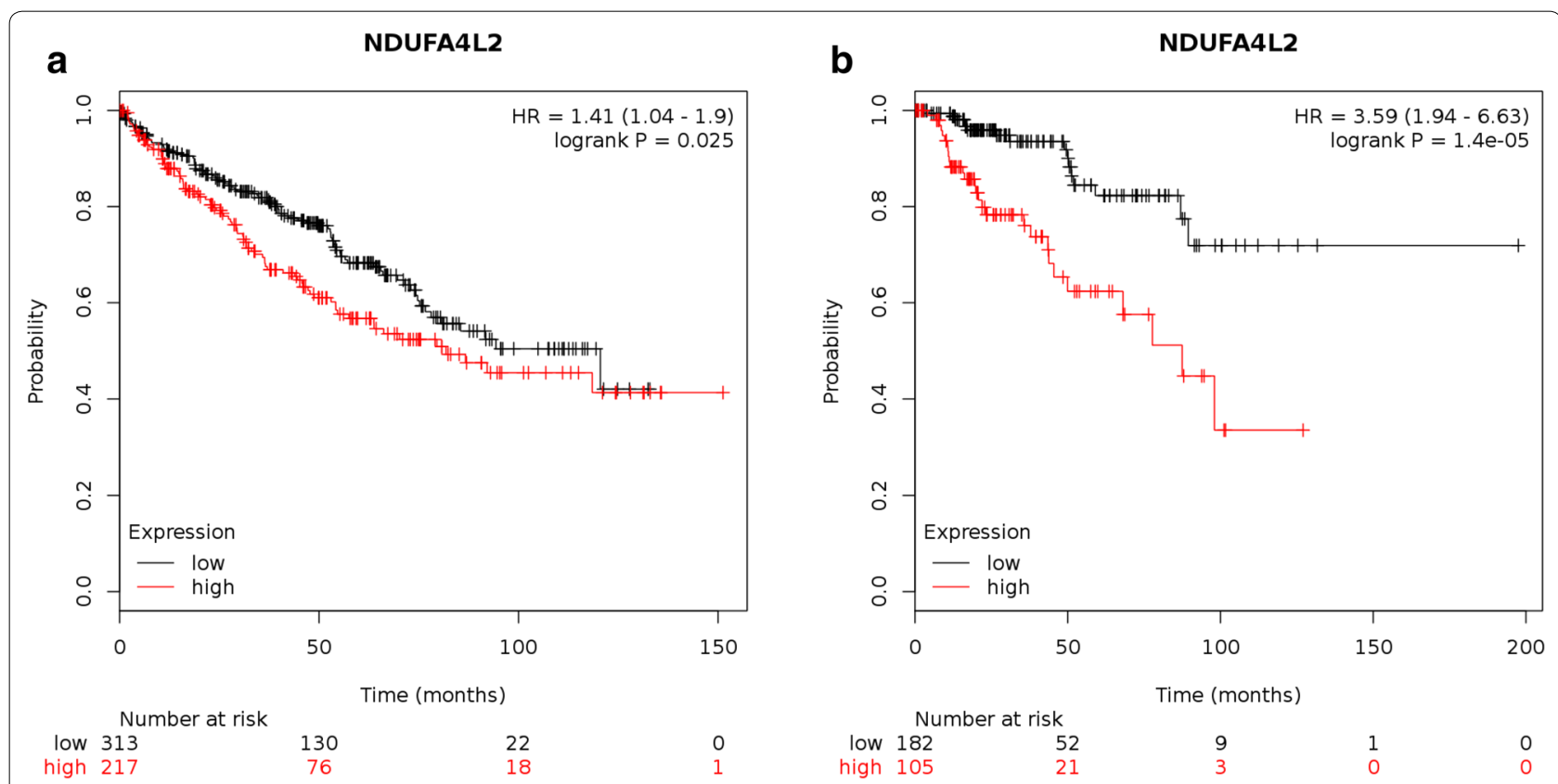

Fig. 7 The prognostic value of NDUFA4L2 in patients with kidney cancer $\mathbf{a}, \mathbf{b}$ overexpressed NDUFA4L2 was associated with worse OS in patients with renal clear cell carcinoma and renal papillary cell carcinoma $(P<0.05$, respectively) 
chemiosmotic coupling, and heat production by uncoupling proteins. and metabolism. For hepatocellular carcinoma, Sarathi et al. found that NDUFA4L2, CRHBP and PIGU were main genes with monotonic changes of expression across cancer stages that are expected to be the therapeutic targets [21]. And in non-small cell lung cancer, some researchers also discovered that mitochondrial NDUFA4L2 protein promotes the vitality of lung cancer cells by repressing oxidative stress, suggesting mitochondrial NDUFA4L2 could represent promising targets for therapy [22]. Unlike SHMT2, NDUFA4L2 had been reported to be involved in the occurrence and development of kidney cancer. Lucarelli et al. identified NDUFA4L2 as the most highly expressed gene in renal cancer cells, which is in accordance with our results. Moreover, their studies suggested that NDUFA4L2 played a critical role in regulating angiogenesis and mitophagy in clear cell renal cell carcinoma [23]. In addition, other validation studies also confirmed that application of NDUFA4L2 in clinical practice could also produce useful effect. Although the number of patients were relatively small, Liu et al. found NDUFA4L2 protein expression was found to be higher in renal clear cell carcinoma tissues 81.4\% (70/86) than in normal tissues $26.7 \%$ (23/86) [24]. Furthermore, NDUFA4L2 overexpressed was reported to be associated with renal clear cell carcinoma malignancy. And its expression was regulated by ELK1 in renal clear cell carcinoma cells [25]. Combined with the above outcomes, we could suppose that SHMT2 and its coexpressed gene NDUFA4L2 may modulate the initiation and development of kidney cancer and influence its prognosis, which may serve as the potential targets for kidney cancer treatment. However, this hypothesis need to be validated by lots of experiments in vivo and vitro.

The limitations of our study included: Firstly, we just validated some findings by polymerase chain reaction and western blot. There are many results need to be verified by multiple experimental methods. Second, the upstream molecule on the roles of SHMT2 in kidney cancer lack of further exploration. It is valued to be explored and verified in the future.

\section{Conclusion}

In summary, SHMT2 and its co-expressed gene NDUFA4L2 were significantly overexpressed in various kinds of kidney cancer. Moreover, high expression of SHMT2 and NDUFA4L2 predicted poor OS in patients with renal clear cell carcinoma. The present study might be benefit for better understanding the clinical significance of SHMT2 and provided a potential therapeutic target for kidney cancer research in the future.

\section{Abbreviations}

SHMT2: Serine Hydroxymethyltransferase 2; ccRCC: Clear cell renal cell carcinoma; NDUFA4L2: NADH dehydrogenase [ubiquinone] 1 alpha sub complex, 4-like 2; GEPIA: Gene Expression Profiling Interactive Analysis; OS: Overall survival.

\section{Acknowledgements}

Not applicable.

\section{Authors' contributions}

HW Collection and/or assembly of data, Manuscript writing; BYL, XSC, WBZ Collection and/or assembly of data. TC Collection and/or assembly of data, Data analysis and interpretation, Manuscript writing, Obtained funding. All authors read and approved the final manuscript.

\section{Funding}

This study was supported by the Health and Family Planning Commission of Hunan Province, People's Republic of China (B2017233).

\section{Availability of data and materials}

The datasets analyzed during the current study are available from the corresponding author on reasonable request.

\section{Ethics approval and consent to participate}

No human or animal specimens were utilized in our studies. And all the datasets were acquired from the public databases and articles, which all met with the Declaration of Helsinki.

\section{Consent for publication}

Not applicable.

\section{Conflicts of interest}

All authors declare that they have no conflict of interests to state.

\section{Author details}

'Department of Urology, Second Affiliated Hospital of Medical School, Xi'an Jiaotong University, Xi'an, Shaanxi 710061, P.R. China. ${ }^{2}$ Urology Department, The First Hospital of Yueyang City, Yueyang City, Hunan Province 414000, China. ${ }^{3}$ Female Urologic Institution, The First Hospital of Yueyang City, Yueyang City, Hunan Province 414000, China.

Received: 5 December 2019 Accepted: 5 October 2020

Published online: 16 October 2020

\section{References}

1. Tahbaz R, Schmid M, Merseburger AS. Prevention of kidney cancer incidence and recurrence: lifestyle, medication and nutrition. Curr Opin Urol. 2018;28(1):62-79. https://doi.org/10.1097/MOU.0000000000000454.

2. Williamson SR, Taneja K, Cheng L. Renal cell carcinoma staging: pitfalls, challenges, and updates. Histopathology. 2019;74(1):18-30. https://doi. org/10.1111/his.13743.

3. Sattler EC, Reithmair M, Steinlein OK. Kidney cancer characteristics and genotype-phenotype-correlations in Birt-Hogg-Dubé syndrome. PLoS ONE. 2018;13(12):e0209504. https://doi.org/10.1371/journal.pone.02095 04.

4. Tani H, Ohnishi S, Shitara H, Mito T, Yamaguchi M, Yonekawa H, Hashizume O, Ishikawa K, Nakada K, Hayashi Jl. Mice deficient in the Shmt2 gene have mitochondrial respiration defects and are embryonic lethal. Sci Rep. 2018;8(1):425. https://doi.org/10.1038/s41598-017-18828-3.

5. Yang X, Wang Z, Li X, Liu B, Liu M, Liu L, Chen S, Ren M, Wang Y, Yu M, Wang B, Zou J, Zhu WG, Yin Y, Gu W, Luo J. SHMT2 Desuccinylation by SIRT5 Drives Cancer Cell Proliferation. Cancer Res. 2018;78(2):372-86. https://doi.org/10.1158/0008-5472.CAN-17-1912.

6. Ning S, Ma S, Saleh AQ, Guo L, Zhao Z, Chen Y. SHMT2 Overexpression predicts poor prognosis in intrahepatic cholangiocarcinoma. Gastroenterol Res Pract. 2018;2018:4369253. https://doi.org/10.1155/2018/43692 53

7. G.S. Ducker, J.M. Ghergurovich, N. Mainolfi, V. Suri, S.K. Jeong, S. Hsin-Jung Li, A. Friedman, M.G. Manfredi, Z. Gitai, H. Kim, J.D. Rabinowitz, Human 
SHMT inhibitors reveal defective glycine import as a targetable metabolic vulnerability of diffuse large B-cell lymphoma, Proc. Natl. Acad. Sci. U.S.A. 114 (43) (2017) 11404-11409, https://doi.org/10.1073/pnas.1706617114.

8. Bernhardt S, Bayerlová M, Vetter M, Wachter A, Mitra D, Hanf V, Lantzsch T, Uleer C, Peschel S, John J, Buchmann J, Weigert E, Bürrig KF, Thomssen C, Korf U, Beissbarth T, Wiemann S, Kantelhardt EJ. Proteomic profiling of breast cancer metabolism identifies SHMT2 and ASCT2 as prognostic factors. Breast Cancer Res. 2017;19(1):112. https://doi.org/10.1186/s1305 8-017-0905-7.

9. Konno M, Asai A, Kawamoto K, Nishida N, Satoh T, Doki Y, Mori M, Ishii H. The one-carbon metabolism pathway highlights therapeutic targets for gastrointestinal cancer (Review). Int J Oncol. 2017;50(4):1057-63. https:// doi.org/10.3892/ijo.2017.3885.

10. Noguchi K, Konno M, Koseki J, Nishida N, Kawamoto K, Yamada D, Asaoka T, Noda T, Wada H, Gotoh K, Sakai D, Kudo T, Satoh T, Eguchi H, Doki Y, Mori M, Ishii H. The mitochondrial one-carbon metabolic pathway is associated with patient survival in pancreatic cancer. Oncol Lett. 2018;16(2):1827-34. https://doi.org/10.3892/ol.2018.8795.

11. Yusenko MV, Kuiper RP, Boethe T, Ljungberg B, van Kessel AG, Kovacs G. High-resolution DNA copy number and gene expression analyses distinguish chromophobe renal cell carcinomas and renal oncocytomas. BMC Cancer. 2009;9:152. https://doi.org/10.1186/1471-2407-9-152.

12. Gumz ML, Zou H, Kreinest PA, Childs AC, Belmonte LS, LeGrand SN, Wu KJ, Luxon BA, Sinha M, Parker AS, Sun LZ, Ahlquist DA, Wood CG, Copland JA. Secreted frizzled-related protein 1 loss contributes to tumor phenotype of clear cell renal cell carcinoma. Clin Cancer Res. 2007;13(16):4740-9. https://doi.org/10.1158/1078-0432.CCR-07-0143.

13. Jones J, Otu H, Spentzos D, Kolia S, Inan M, Beecken WD, Fellbaum C, Gu X, Joseph M, Pantuck AJ, Jonas D, Libermann TA. Gene signatures of progression and metastasis in renal cell cancer. Clin Cancer Res. 2005:11(16):5730-9. https://doi.org/10.1158/1078-0432.CCR-04-2225.

14. Beroukhim R, Brunet JP, Di Napoli A, Mertz KD, Seeley A, Pires MM, Linhart D, Worrell RA, Moch H, Rubin MA, Sellers WR, Meyerson M, Linehan WM, Kaelin WG, Signoretti S. Patterns of gene expression and copy-number alterations in von-hippel lindau disease-associated and sporadic clear cell carcinoma of the kidney. Cancer Res. 2009;69(11):4674-81. https://doi. org/10.1158/0008-5472.CAN-09-0146.

15. Li AM, Ducker GS, Li Y, Seoane JA, Xiao Y, Melemenidis S, Zhou Y, Liu L, Vanharanta S, Graves EE, Rankin EB, Curtis C, Massagué J, Rabinowitz JD, Thompson CB, Ye J. Metabolic profiling reveals a dependency of human metastatic breast cancer on mitochondrial serine and one-carbon unit metabolism. Mol Cancer Res. 2020:18(4):599-611. https://doi. org/10.1158/1541-7786.MCR-19-0606.

16. D. Kim, B.P. Fiske, K. Birsoy, E. Freinkman, K. Kami, R.L. Possemato, Y. Chudnovsky, M.E. Pacold, W.W. Chen, J.R. Cantor, L.M. Shelton, D.Y. Gui, M. Kwon, S.H. Ramkissoon, K.L. Ligon, S.W. Kang, M. Snuderl, M.G. Vander Heiden, D.M. Sabatini, SHMT2 drives glioma cell survival in ischaemia but imposes a dependence on glycine clearance, Nature 520 (7547) (2015) 363-367, https://doi.org/10.1038/nature14363.
17. A. Rai, D.W. Greening, M. Chen, R. Xu, H. Ji, R.J. Simpson, Exosomes Derived from Human Primary and Metastatic Colorectal Cancer Cells Contribute to Functional Heterogeneity of Activated Fibroblasts by Reprogramming Their Proteome, Proteomics (2018) e1800148, https:// doi.org/10.1002/pmic.201800148.

18. Nilsson R, Jain M, Madhusudhan N, Sheppard NG, Strittmatter L, Kampf C, Huang J, Asplund A, Mootha VK. Metabolic enzyme expression highlights a key role for MTHFD2 and the mitochondrial folate pathway in cancer. Nat Commun. 2014;5:3128. https://doi.org/10.1038/ncomms4128.

19. Ye J, Fan J, Venneti S, Wan YW, Pawel BR, Zhang J, Finley LW, Lu C, Lindsten T, Cross JR, Qing G, Liu Z, Simon MC, Rabinowitz JD, Thompson CB. Serine catabolism regulates mitochondrial redox control during hypoxia. Cancer Discov. 2014;4(12):1406-17. https://doi.org/10.1158/2159-8290. CD-14-0250.

20. Wei Z, Song J, Wang G, Cui X, Zheng J, Tang Y, Chen X, Li J, Cui L, Liu CY, Yu W. Deacetylation of serine hydroxymethyl-transferase 2 by SIRT3 promotes colorectal carcinogenesis. Nat Commun. 2018;9(1):4468. https //doi.org/10.1038/s41467-018-06812-y.

21. Sarathi A, Palaniappan A. Novel significant stage-specific differentially expressed genes in hepatocellular carcinoma. BMC Cancer. 2019;19(1):663. https://doi.org/10.1186/s12885-019-5838-3.

22. Meng L, Yang $X$, Xie X, Wang M. Mitochondrial NDUFA4L2 protein promotes the vitality of lung cancer cells by repressing oxidative stress. Thorac Cancer. 2019;10(4):676-85. https://doi.org/10.1111/17597714.12984.

23. G. Lucarelli, M. Rutigliano, F. Sallustio, D. Ribatti, A. Giglio, M. Lepore Signorile, V. Grossi, P. Sanese, A. Napoli, E. Maiorano, C. Bianchi, R.A. Perego, M. Ferro, E. Ranieri, G. Serino, L.N. Bell, P. Ditonno, C. Simone, M. Battaglia, Integrated multi-omics characterization reveals a distinctive metabolic signature and the role of NDUFA4L2 in promoting angiogenesis, chemoresistance, and mitochondrial dysfunction in clear cell renal cell carcinoma, Aging (Albany NY) 10 (12) (2018) 3957-3985, https://doi. org/10.18632/aging.101685.

24. Liu L, Lan G, Peng L, Xie X, Peng F, Yu S, Wang Y, Tang X. NDUFA4L2 expression predicts poor prognosis in clear cell renal cell carcinoma patients. Ren Fail. 2016;38(8):1199-205. https://doi.org/10.1080/08860 22X.2016.1208517.

25. Wang L, Peng Z, Wang K, Qi Y, Yang Y, Zhang Y, An X, Luo S, Zheng J. NDUFA4L2 is associated with clear cell renal cell carcinoma malignancy and is regulated by ELK1. PeerJ. 2017;5:e4065. https://doi.org/10.7717/ peerj.4065.

\section{Publisher's Note}

Springer Nature remains neutral with regard to jurisdictional claims in published maps and institutional affiliations.

\footnotetext{
Ready to submit your research? Choose BMC and benefit from:

- fast, convenient online submission

- thorough peer review by experienced researchers in your field

- rapid publication on acceptance

- support for research data, including large and complex data types

- gold Open Access which fosters wider collaboration and increased citations

- maximum visibility for your research: over $100 \mathrm{M}$ website views per year
}

At BMC, research is always in progress.

Learn more biomedcentral.com/submissions 